\title{
Evaluación de Procesos Participativos para la Formación Ambiental a partir del Conocimiento de Watsimba Tigridia pavonia (L.F.) DC. en Sibundoy, Putumayo, Colombia.
}

\author{
Hugo F. Leonel y Gloria C. Luna-Cabrera \\ Universidad de Nariño, Facultad de Ciencias Agrícolas, Programa de Ingeniería Agroforestal, Calle 18 \\ Carrera 50 Ciudadela Universitaria Torobajo, Pasto, Nariño, Colombia. (e-mail: hleonel2001@gmail.com; \\ grupopifil@gmail.com)
}

Recibido Jun. 3, 2016; Aceptado Ago. 3, 2016; Versión final Sep. 30, 2016, Publicado Feb. 2017

\begin{abstract}
Resumen
Se evaluó los niveles de participación en la construcción de conocimiento agronómico y usos de Watsimba Tigridia pavonia (L.F.) DC., en Sibundoy, Putumayo, Colombia. Mediante metodología de investigación acción participativa, con técnicas y herramientas para toma de datos, visitas a treinta chagras y entrevistas semiestructuradas, se determinaron niveles de participación en categorías nulo-bajo, medio y alto. Los indígenas presentaron nivel Alto de participación en conocimiento previo de usos de T. pavonia. Después de los procesos participativos implementados para la formación ambiental, los mayores niveles de distribución porcentual de conocimientos científicos adquiridos sobre usos y aspectos agronómicos fueron la Escuela normal Superior Putumayo y la Universidad de Nariño. Se concluye que las técnicas comunicacionales y procesos de aprendizaje empleados contribuyeron a la valoración y rescate de la especie, incrementando el nivel de participación de los diferentes actores.
\end{abstract}

Palabras clave: especies promisorias; Watsimba; flor de tigre; participación

\section{Evaluation of Participatory Processes for Environmental Training based on the Knowledge of Watsimba Tigridia pavonia (L.F.) DC., in Sibundoy, Putumayo, Colombia}

\begin{abstract}
Levels of participation in the construction of agronomic knowledge and uses of Watsimba Tigridia pavonia (L.F.)DC., in Sibundoy, Putumayo, Colombia were evaluated. Through research methodology employing participatory action of with techniques and tools to the data recollection, thirty chagras were visited and semi-structured interviews were taken to determinate different levels of participation in three categories: null-low, average and high. Native Indians presented high level of knowledge on uses of $T$. pavonia. After the participatory processes implemented for the environmental training the highest levels of scientific knowledge acquisition on uses and agronomic aspects were the Higher Education School of Putumayo and the University of Nariño. As a conclusion, the communication techniques and learning processes used contributed to the valuation and rescuing of the species, increasing the level of participation of the different actors involved.
\end{abstract}




\section{INTRODUCCIÓN}

La palabra participar viene del latín participare (tomar parte en algo), compuesta de pars, partis (parte) y el verbo capere (tomar, agarrar), pero se caracteriza por ser muy amplio, equívoco, ambiguo, polisémico, relativo y con fuertes connotaciones ideológicas (Sánchez y Del Pino, 2008); busca un objetivo específico como informarse, tomar parte en una decisión, involucrarse en alguna discusión, integrarse, o simplemente beneficiarse de la ejecución y solución de un problema puntual (Leonel, 2011) además de obtener dominio de sus propias vidas (Sánchez y del Pino, 2008), contribuye en la construcción de una sociedad alternativa (Dubois, 2008); refuerza la negociación, el diálogo y la confianza mutua (Hatcher, 2009), el trabajo en equipo, el bien común y la generación de conocimiento.

La participación se convierte en uno de los pilares fundamentales para la generación de conocimiento interdisciplinar (García, et al., 2008), como factor clave de ventaja competitiva, eficaz, oportuno con el cambio dinámico del entorno y en procesos de gestión ambiental que conllevan a acciones de diálogo, decisiones y consensos (Leonel, et al., 2010) en la construcción socio-ambiental de una realidad; en donde es de gran importancia la formación ambiental por medio de aprendizajes significativos. En este sentido, es indispensable que quien participe, tenga los conocimientos previos y el interés en relacionarlos o incorporarlos para su propio bienestar y el de los demás, permitiendo su desarrollo como individuo e integrante de una sociedad con enfoques holísticos e interdisciplinarios.

Los enfoques holísticos e interdisciplinarios para la comprensión de la naturaleza, la conservación y la sostenibilidad de sistemas acoplados a lo humanos son tratados con frecuencia como sistemas socioecológicos (SES), es decir, sistemas adaptativos complejos con características claves tales como: procesos socioculturales biogeofísicos integrados, autorganización, dinámicas no lineales retroalimentación entre procesos sociales y ecológicos, cambios espacio temporales, propiedades emergentes y la imposibilidad de extrapolar información de una SES a otra (Delgado et al., 2015).

En la investigación, a pesar de los diferentes usos de $T$. pavonia dados por la comunidad indígena Inga y Camentsa y su importancia en el Alto Putumayo, Colombia, aún su conocimiento es limitado y por ende su abordaje interdisciplinar. El género Tigridia, presenta aproximadamente 35 especies que se distribuyen desde Chile hasta México. Es una planta propia de clima templado, se encuentra en varias regiones del mundo como Tasmania, Geelong; pero es nativa de México. En Colombia se encuentra en el departamento de Cundinamarca, los alrededores de Popayán, la Bota Caucana y el Valle de Sibundoy donde no tiene un manejo específico sino ocasionalmente en huertos familiares (De Villota, 2010). Es poco el conocimiento científico de la especie y se está perdiendo el ancestral, ya que los colonos y jóvenes la consideran como un arvense que invade sus chagras (huertos), además existe escasa participación de los diferentes actores locales para su conservación.

Esta investigación se enfocó en la evaluación de procesos participativos para la formación ambiental a partir del conocimiento de T. pavonia, en aspectos agronómico y de usos; a partir de la metáfora que asemeja a ésta con una escalera, donde cada peldaño o escalón corresponde a un nivel de participación y representa a este fenómeno social como un proceso gradual y ascendente de empoderamiento.

\section{METODOLOGÍA}

Este estudio fue realizado en el municipio de Sibundoy, situado entre los $766^{\circ} 55^{\prime} 12^{\prime \prime}$ de longitud Oeste y 011ㄱ $26^{\prime \prime}$ de latitud Norte a una altura sobre el nivel del mar de $2224 \mathrm{~m}$; con temperatura promedio de 15드. humedad relativa de $83 \%$ y precipitación anual de $1715 \mathrm{~mm}$ (IDEAM, 2007, citado por Paguatian, 2007). Pertenece a la zona de vida bosque muy húmedo montano bajo (bmh - MB). La base de la economía del municipio se soporta en el sector primario o agropecuario y en el terciario o de servicios, siendo el comercio uno de los de mayor crecimiento. La investigación se desarrolló con el grupo los Exploradores de la Escuela Normal Superior del Putumayo - ENSUP, municipio de Sibundoy, conformado por 16 estudiantes de 8 a 18 años, de los distintos grados escolares; además se trabajó con 20 fincas de campesinos e indígenas del grupo Camentsa y estudiantes tesistas de los programa de Ingeniería Agroforestal y Agronómica de la Universidad de Nariño.

El enfoque metodológico se enmarca dentro de la investigación acción participativa (Fals, 2012), donde los diferentes actores generan y hacen aprehensión del conocimiento, a partir del intercambio de saberes y son los responsables de sus propias decisiones. Mediante la alianza entre la UDENAR (grupo de investigación PIFIL) y la ENSUP (grupo Los Exploradores), se conoció el estado del arte del avance en el conocimiento de T. pavonia, tanto a nivel empírico como científico; para lo cual se realizaron 30 visitas a fincas o chagras con presencia de T. pavonia. Durante la investigación participativa, se llegó al consenso de evaluar a partir de un proceso de educación ambiental, para lo cual se plantearon unas fases. Las fases de la Figura 1 
fueron distribuidas en tres categorías de análisis (conocimiento agronómico, usos y niveles de participación). La evaluación en cuanto a conocimientos agronómicos y usos, se desarrolló a partir de un instrumento que contenía preguntas relacionadas con las variables, aplicado al principio de la investigación y al final del proceso. Las respuestas fueron categorizadas en porcentajes de acuerdo al número de personas por tipo de actor (UDENAR, ENSUP, indígenas y campesinos) que contestaban bien o mal de acuerdo a la siguiente escala de valoración: conocimiento Nulo o Bajo (0-33\% de personas), medio (34-65\%) y alto (66-100\%).

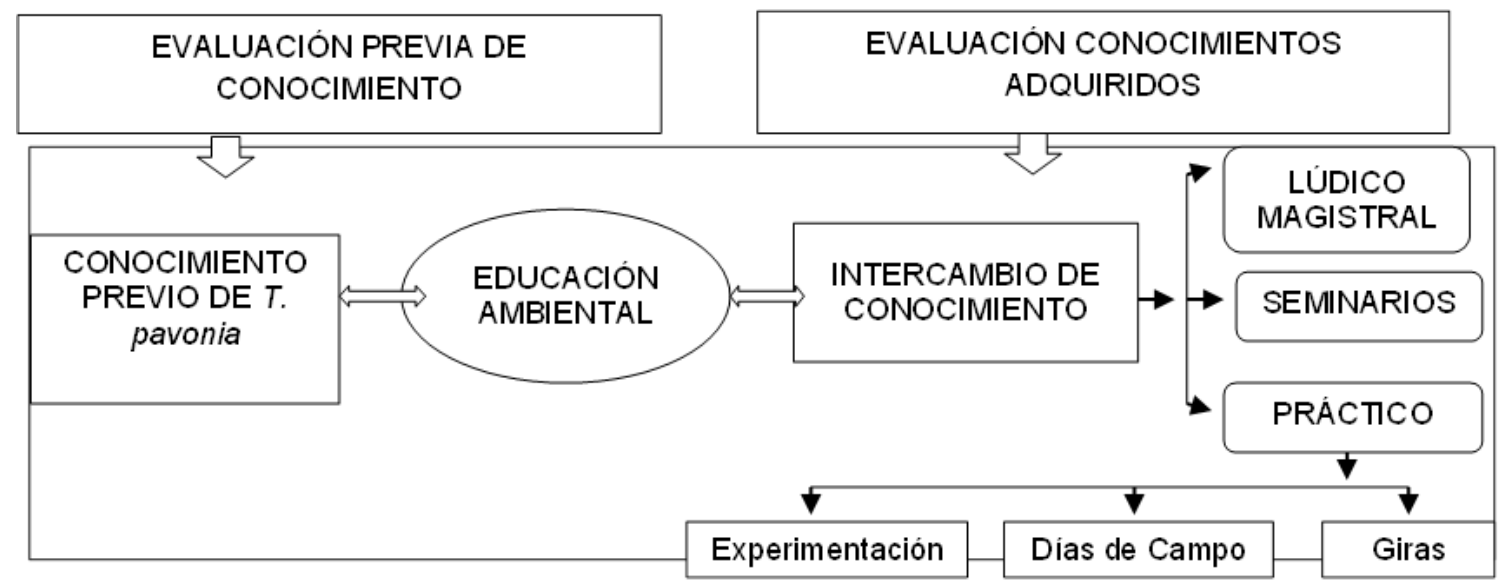

Fig. 1: Estrategia participativa de investigación para generación de conocimiento de Tigridia pavonia (L.F.) DC.

Se evaluó la participación de cada uno de los actores involucrados por medio de la metodología propuesta por (Geilfus, 1997, citado por Leonel, 2011), utilizando siete peldaños de la escalera de participación: 1) Pasividad: las personas participan cuando se les informa; no tienen ninguna incidencia en las decisiones y la implementación del proyecto; 2) Suministro de información: las personas participan respondiendo a encuestas; no tiene posibilidad de influir ni siquiera en el uso que se va a dar de la información; 3) Participación por consulta: las personas son consultadas por agentes externos que escuchan su punto de vista, esto sin tener incidencia sobre las decisiones que se tomarán a raíz de dichas consultas; 4) Participación por incentivos: las personas participan proveyendo principalmente trabajo a otros recursos (tierra para ensayos), a cambio de ciertos incentivos (materiales, sociales, capacitación); sin embargo, no tienen incidencia directa en las decisiones; 5) Participación funcional: las personas participan formando grupos de trabajo para responder a objetivos predeterminados por el proyecto, no tienen incidencia sobre la formulación, pero se los toma en cuenta en el monitoreo y el ajuste de actividades; 6) Participación interactiva: los grupos locales organizados participan en la formulación, implementación y evaluación del proyecto; lo que implica procesos de enseñanza-aprendizaje, sistemáticos y estructurados, y la toma de control en forma progresiva del proyecto; y finalmente; y 7) Auto-desarrollo: los grupos locales organizados toman iniciativas sin esperar intervenciones externas; las intervenciones se hacen en forma de asesoría y como socios.

El nivel de participación se caracterizó como "Nulo-bajo", "Medio" y "Alto", propuesto por Leonel (2011), en donde: a) Participación de nivel "Nulo-Bajo": aquí se incluyeron a los actores que no participaron, solamente asistían a las reuniones o simplemente eran informados; es decir, estaban en los peldaños de "pasividad", "suministro de información" y "participación por consulta"; b) Participación de nivel "Medio": se incluyeron a quienes colaboraron con dinero, o realizaron trabajos a cambio de ciertos incentivos, pero no tuvieron incidencia directa en las decisiones; es decir, estuvieron en los peldaños "participación por incentivos" y "participación funcional"; c) Participación de nivel "Alto": se incluyeron a todos los individuos que tuvieron incidencia directa en las decisiones, ocupaban un cargo directivo, solicitaban información y hacían denuncias; es decir, los que estaban en los peldaños más altos de la escalera: "participación interactiva" y "auto-desarrollo".

\section{RESULTADOS Y DISCUSIÓN}

De acuerdo a la investigación acción participativa, los resultados se resumen en las Tablas 1,2, 3 y Figura $2 a$ y $2 b$, según categoría de análisis.

\section{1) Conocimientos agronómicos}

Los conocimientos previos de ENSUP, comunidades indígenas e investigaciones de la UDENAR en usos y aspectos agronómicos de la especie facilitaron la aprehensión del conocimiento; el cual, está relacionado 
directamente con el nivel de participación de los actores en las diferentes actividades, logrando así participación funcional, interactivo y de autodesarrollo, Tabla 1; además de identificar los usos que las comunidades le han dado históricamente a la especie. Pérez et al., (2015), manifiestan que los agricultores tienen la oportunidad de aprender e implementar habilidades de pensamiento crítico, sistematizar y ampliar el conocimiento local de la vegetación, que según Torregroza et al., (2014), se constituye en un aspecto importante en términos de gestión, al ser capaz de manifestar el valor diferencial que le otorgan a cada especie o individuo, evidenciándose el nivel de identificación, preferencia y afectividad social.

Tabla 1: Matriz de conocimiento agronómico local y científico de T. pavonia, municipio de Sibundoy, Putumayo.

\begin{tabular}{|l|l|l|}
\hline \multirow{2}{*}{ Generalidades } & \multicolumn{2}{|c|}{ Lonocimiento } \\
\cline { 2 - 3 } & \multicolumn{1}{|c|}{ Científico } \\
\hline Propagación & Por semilla y por bulbo & In vitro por meristemos \\
\hline Siembra & Regeneración natural & Distancias de siembra $50 \times 50 \mathrm{~cm}$ \\
\hline Fertilización & $\begin{array}{l}\text { No se fertiliza, se considera } \\
\text { maleza }\end{array}$ & Abono orgánico \\
\hline Crecimiento & mejor crecimiento bajo sombra & Altura promedio $60 \mathrm{~cm}$ \\
\hline Manejo fitosanitario & No identificado & No investigado \\
\hline Cosecha & $6-7$ meses & A los 7 meses \\
\hline $\begin{array}{l}\text { Potencial } \\
\text { agroforestal }\end{array}$ & No se conoce & $\begin{array}{l}\text { Estudios preliminares en asocio laurel de cera+ } \\
\text { maíz+ Watsimba Laurel de cera con Watsimba }\end{array}$ \\
\hline
\end{tabular}

Los resultados muestran que el conocimiento agronómico local de $T$. pavonia es limitado con respecto al científico, lo cual puede obedecer a que las comunidades indígenas y campesinas no la cultivan, ni la manejan porque la consideran como una arvense. Rodríguez et al., (2012), manifiestan que se deben fomentar estrategias de cambio de pensamiento, actuación y sentimiento que sustituyan los actuales valores individualistas sin olvidar que las personas no son simples agentes pasivos del cambio que construyen su realidad a partir de intercambios comunicacionales. El conocimiento ancestral de $T$. pavonia en la fase inicial de la investigación, comparado con el conocimiento científico adquirido un año después del proceso de formación ambiental en el municipio de Sibundoy Putumayo se detallan en las figuras $2 \mathrm{a}$ y $2 \mathrm{~b}$. Por medio de las técnicas comunicacionales empleadas en los recorridos de campo, se identificaron los usos que la comunidad le da a T. pavonia (Tabla 2).

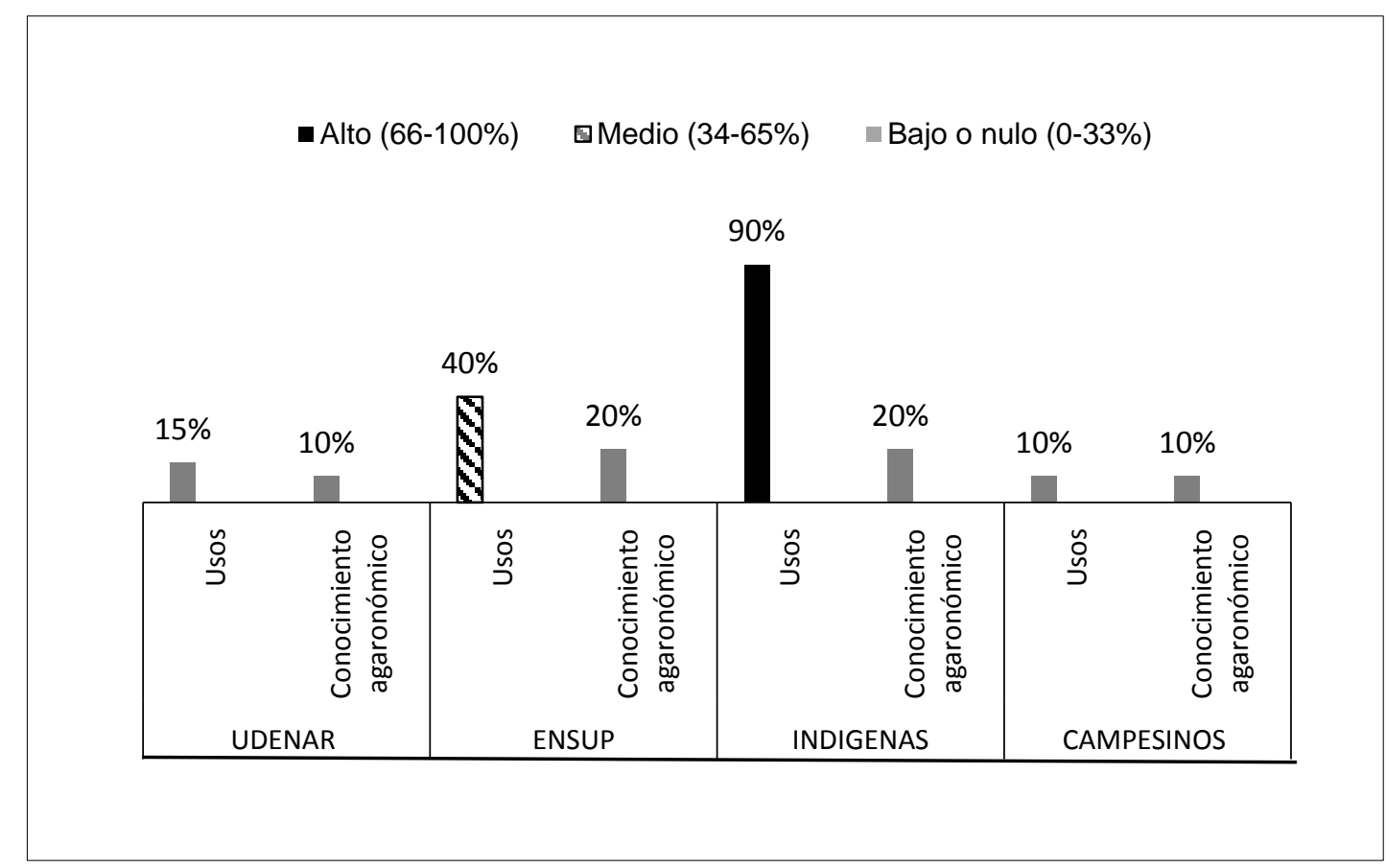

Fig. 2a: Distribución porcentual del nivel conocimiento previo de los actores, en cuanto a usos y conocimiento agronómico 


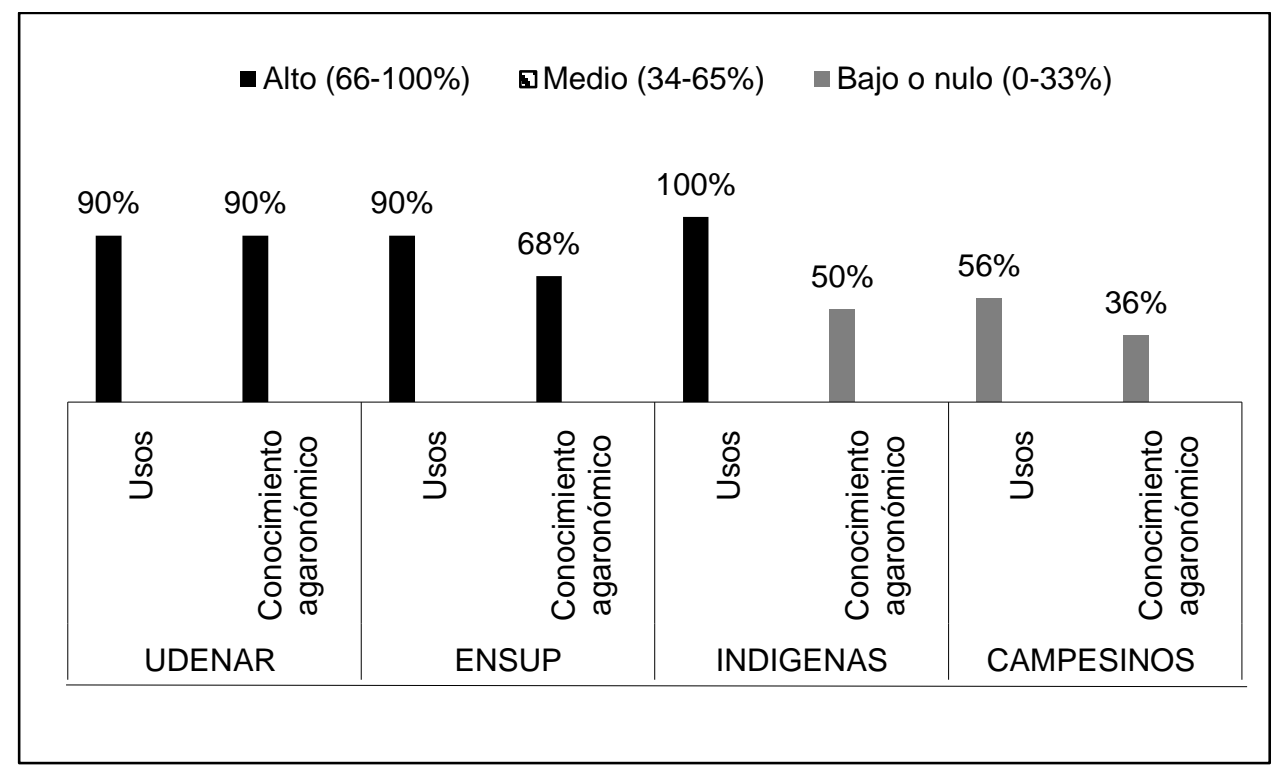

Fig. 2b: Distribución porcentual de conocimiento científico adquirido después de la investigación

Las figuras muestran un incremento en la adquisición de conocimientos científicos, lo cual se ve reflejado por la intervención de las diferentes instituciones, quienes después de las investigaciones realizadas lograron resultados positivos, lo cual puede obedecer a lo manifestado por Urquiza y Cadenas (2015), que estas relaciones permiten paralelamente aprovechar el conocimiento de las comunidades que están en directa relación con el medio ambiente. Con lo anterior cabe resaltar la importancia de los procesos participativos en donde diferentes instituciones $u$ organizaciones pueden contribuir a mejorar los conocimientos y prácticas dentro de una comunidad, tal como lo manifiesta Fernández y Gutiérrez (2013), para quien la revalorización de las actitudes y conductas ancladas en valores éticos posibilitan la participación de la ciudadanía en la defensa de bienes sociales, culturales, económicos, naturales que constituyen el acervo socio-histórico y cultural de los humanos y de su medio ambiente.

\section{2) Usos de Tigridia pavonia (L.F.) DC}

T. pavonia, tradicionalmente se ha mantenido en las chagras como unos de los componentes básicos para la alimentación, empleando el bulbo en diferentes preparaciones culinarias como elaboración de galletas, panderos, biscochos y tortas, entre otros; el bulbo desgajado se deshidrata para freír y obtener un pasaboca de gran aceptación por los habitantes del alto Putumayo, denominado "Watsifri". Martínez et al., (2007), plantean que el bulbo cocinado es utilizado como alimento; por otra parte, (Piña et al., 2010a), afirman que esta especie es usada como ornamental, alimento y con fines medicinales; además tiene un alto potencial económico. En el uso artesanal se resalta la inmortalización de la flor para uso en diferentes decoraciones y se ha explorado su utilización en bisutería (De Villota, 2010, charla personal). En el uso de colorantes, la flor, tienen un potencial para la elaboración de pintura artística; sin embargo esto no se ha evaluado aún.

Tabla 2: Matriz de los usos de la T. pavonia, Sibundoy, Putumayo, estudio con base en Arias y Cárdenas (2007)

\begin{tabular}{|c|c|c|c|c|c|}
\hline \multirow[b]{2}{*}{ Categoría } & \multirow[b]{2}{*}{ Descripción } & \multicolumn{3}{|c|}{ Nivel de aprovechamiento } & \multirow{2}{*}{$\begin{array}{l}\text { Parte de la } \\
\text { especie que se } \\
\text { aprovecha }\end{array}$} \\
\hline & & Alto & Medio & Bajo & \\
\hline Alimento & $\begin{array}{l}\text { Plantas aprovechadas para el consumo } \\
\text { humano. }\end{array}$ & & $\mathrm{x}$ & & Bulbo \\
\hline Artesanal & $\begin{array}{l}\text { Especies, fibras para cestería, pulpa para } \\
\text { elaboración artesanal de papel, semillas y/o } \\
\text { recipientes. }\end{array}$ & & & $\mathrm{x}$ & Flor \\
\hline Colorante & $\begin{array}{l}\text { Usada para obtener tintes naturales, } \\
\text { colorantes. }\end{array}$ & & & $\mathrm{x}$ & Flor \\
\hline Cultura & Utilizada en actividades sociales o rituales. & & $\mathrm{x}$ & & Planta \\
\hline Forraje & Sirve para alimento animal. & & & $\mathrm{x}$ & Bulbo \\
\hline Medicinal & Usada para tratar o prevenir enfermedades. & & $\mathrm{x}$ & & Bulbo \\
\hline Ornamental & $\begin{array}{l}\text { Con uso actual o potencial en el ornato y } \\
\text { decoración de espacios. }\end{array}$ & $\mathrm{x}$ & & & Planta \\
\hline
\end{tabular}


El uso ornamental se da por la vistosidad de sus flores. Piña et al., (2010b), afirman que es una planta bulbosa con alto potencial ornamental y fue una de las plantas favoritas en los jardines de los gobernantes aztecas; se encuentra ampliamente distribuida en diferentes países de Europa, Asia y Australia, donde es comercializada como planta ornamental (Vázquez, et al., 2001a); ya que por su vistosidad es usada como ornamental (Piña et al., 2010b). Sin embargo, Vázquez et al., (2001b), mencionan que el mal manejo de la especie puede ocasionar erosión genética.

Dentro de las creencias populares alrededor de esta planta (De Villota, 2005, citado por Paguatian, 2007), mencionan: Según los Camëntsa, la $T$. pavonia, fue la planta que los salvó de la hambruna, en tiempos en los que el Valle de Sibundoy fue azotado por una fuerte helada que destruyó gran parte de sus cultivos. En la región los indígenas la consideran como regalo de Dios para los pobres, quienes no pueden comprar otros alimentos como la papa y manifiestan que es una planta que no requiere de ser abonada y la consideran mágica por tener los colores del poder. En Guateque-Cundinamarca, afirman que las flores producen pecas en la piel, además el sembrarla cerca de sus casas los libra de maleficios. Luis Jajoy, habitante del municipio de Sibundoy, manifiesta "La planta flor de un día, fue utilizada por mis abuelos, quienes nos enseñaron a comerla, también la usamos para que nuestra casa se vea más bonita y aleja los maleficios".

La planta es introducida a la región por medio de la cultura que desciende del imperio Inca, difundiendo la especie a los Camëntsa, con quienes comparten el territorio, los antepasados indígenas fueron quienes le asignaron el nombre de Watsimba que significa" planta silvestre" por la facilidad con que crece y se reproduce, razón por la cual se ha mantenido en la región Amazónica (De Villota (2005), citado por Paguatian, 2007:19). Es así, como Nancy Mavisoy Muchavisoy, habitante del municipio de Sibundoy, manifiesta: "La planta crece mejor donde le llega la sombra y hay humedad... se encuentra también dentro de los bosques".

Las investigaciones realizadas por el grupo los Exploradores-ENSUP han arrojado los siguientes resultados: con el bulbo se preparan brebajes que se utilizan como sueros de hidratación oral, en pacientes que sufren de insomnio se pela el bulbo, se muele y después se cocina, el agua que se obtiene con esa preparación se bebe con azúcar al gusto. En personas que manejan grandes niveles de estrés, se ingiere el bulbo cocido sin sal. Para la bronquitis, dolores de cabeza, y dolores menstruales se usa la hoja machacada en infusión. Algunas campesinas la utilizan para tratar la esterilidad de las vacas y el jarabe de la Watsimba roja se utiliza mucho para los problemas bronquiales y pulmonares y el extracto de las flores para problemas de venas varices y formación de trombos que ocasionan flebitis.

\section{3) Niveles de participación}

Considerada la capacitación como escenario para el intercambio de saberes, articulada con actividades lúdicas, a través de aprovechamiento del juego como eje dinamizador de la enseñanza-aprendizaje, se facilitó la participación activa, crítica y propositiva de los asistentes a los talleres y afianzó los sentimientos comunitarios. Leonel et al., (2013); Leonel y Luna (2016), plantean que el juego, en especial el tradicional revela a que cultura social pertenece el sujeto y, la noción de cohesión social cobra todo su sentido y espacio; privilegiándose una concepción del mismo sujeto, la cultura, el enseñar y el aprender, sobre: la naturaleza, la comunicación y el sentido de conocimiento. El juego como instrumento pedagógico, actúa en dos sentidos, por un lado construye elementos cognitivos y por otro desarrolla aspectos afectivos, componentes que se complementan en la visión integral del ser humano (Holguin y Lezaca, 2010). Los juegos pueden ser diseñados para facilitar tanto la exploración y el modo performativo de acción (Linderoth, 2012), forman parte importante en el aprendizaje, pues logran conectar el nuevo conocimiento con el existente, modificando su estructura cognitiva (León et al., 2014).

La influencia entre la conducta y el conocimiento de los individuos, facilitó la participación de los actores en la investigación y la identificación y selección de las temáticas de capacitación (Tabla 3). Pérez, et al., (2015), manifiestan que con la discusión y el trabajo en grupo los participantes son incluidos mediante el aprovechamiento de su conocimiento individual, son estimulados para compartir sus saberes, adquiridos a través de años de experiencia. En la Tabla 3, se evidencia que el mayor peso en los procesos de participación se concentra en el "Nivel Medio", liderados por las Instituciones Educativas; marginando el liderazgo de las comunidades indígenas y campesinas; situación que cambia en los recorridos de campo, donde estos actores demuestran conocimientos ecológicos y diversidad de usos de la especie, además de ser los generadores del conocimiento histórico de la misma. Mediante el diálogo de saberes, estos espacios de conocimiento se enriquecen y fortalecen en la medida que todo el equipo de trabajo y actores aportan sus ideas a fin de lograr innovadoras propuestas que sin perder su esencia de profundidad en los temas técnicos, facilitan la interacción entre los participantes, generan motivación constante, diviertan y animen a la acción y alcance de un mediano plazo a la apropiación social del conocimiento (Leonel y Luna, 2016). 
Tabla 3: Niveles de participación de los actores identificados para la generación de conocimiento de Tigridia pavonia (L.F.) DC., Sibundoy, Putumayo.

\begin{tabular}{|c|c|c|c|c|}
\hline \multirow{2}{*}{ Actividad } & \multirow{2}{*}{ Tipo de Actor } & \multicolumn{3}{|c|}{ Nivel de participación } \\
\hline & & Nulo - bajo & Medio & Alto \\
\hline \multirow{4}{*}{$\begin{array}{l}\text { Identificación de temas de } \\
\text { capacitación }\end{array}$} & UDENAR & & & Autodesarrollo \\
\hline & ENSUP & & & Autodesarrollo \\
\hline & Indígenas & Información & & \\
\hline & Campesinos & Información & & \\
\hline \multirow[t]{4}{*}{ Capacitaciones } & UDENAR & & & Interactiva \\
\hline & ENSUP & & & Interactiva \\
\hline & Indígenas & & Funcional & \\
\hline & Campesinos & & Funcional & \\
\hline \multirow[t]{4}{*}{ Recorridos de campo } & UDENAR & & & Interactiva \\
\hline & ENSUP & & & Interactiva \\
\hline & Indígenas & & & Interactiva \\
\hline & Campesinos & & & Interactiva \\
\hline \multirow[t]{4}{*}{ Usos de la especie } & UDENAR & & Funcional & \\
\hline & ENSUP & & Funcional & \\
\hline & Indígenas & & & Interactiva \\
\hline & Campesinos & Información & & \\
\hline \multirow{4}{*}{$\begin{array}{l}\text { Reconstrucción histórica } \\
\text { de la especie }\end{array}$} & UDENAR & & Funcional & \\
\hline & ENSUP & & Funcional & \\
\hline & Indígenas & & & Autodesarrollo \\
\hline & Campesinos & & Funcional & \\
\hline \multirow[t]{4}{*}{ Conocimiento agronómico } & UDENAR & & & Autodesarrollo \\
\hline & ENSUP & & Funcional & \\
\hline & Indígenas & Informativo & & \\
\hline & Campesinos & Informativo & & \\
\hline \multirow[t]{4}{*}{ Conocimiento ecológico } & UDENAR & & & Autodesarrollo \\
\hline & ENSUP & & Funcional & \\
\hline & Indígenas & & Funcional & \\
\hline & Campesinos & & Funcional & \\
\hline \multirow{4}{*}{$\begin{array}{l}\text { Conocimiento } \\
\text { agroindustrial }\end{array}$} & UDENAR & & Funcional & \\
\hline & ENSUP & & & Autodesarrollo \\
\hline & Indígenas & & Funcional & \\
\hline & Campesinos & & Funcional & \\
\hline
\end{tabular}

Lo anterior señala que cuando se tiene conocimiento de algo y las personas se sienten identificadas con los procesos de toma de decisiones, se potencializa la participación, llegando hasta el autodesarrollo; lo cual, corrobora lo planteado por Leonel et al., (2010), quienes afirman que la participación tiene que ver con la construcción social de una realidad dada que conlleve a acciones de diálogo, decisiones y consensos; sin embargo, no se pueden generar aprendizajes significativos, si no cuenta en su estructura cognoscitiva con los conocimientos previos necesarios y dispuestos (activados) para enlazar los nuevos aprendizajes propuestos (Domínguez, 2011) que aporten al cambio social; en tanto los protagonistas, sujetos significantes y significadores interactúan en prácticas socio culturales concretas, que desatan procesos de comunicación (Villamayor, 2006).

Otro aspecto no menos importante es el carácter dinámico de la participación ya que no se trata de conocimientos ancestrales invariables sino de saberes que se fueron generando en diferentes épocas y que han sobrevivido gracias a que continuaron siendo usados a través del tiempo y modificados a diferentes necesidades y condiciones del entorno (Endere, 2013), de ahí la importancia de la recuperación del conocimiento agronómico y de usos de T. pavonia, en donde los protagonistas principales son los actores locales, de tal forma que se cambie el sistema tradicional de estructura de desarrollo que desconoce el conocimiento empírico resultado de la valoración y apropiación de especies vegetales que fortalecen el tejido social y las relaciones socio afectivas con la naturaleza. 


\section{CONCLUSIONES}

El enfoque de formación ambiental e implementación de técnicas de observación, experimentación, investigación, implementación de lúdica y articulación de las instituciones contribuyeron en el incremento de los niveles de participación de los diferentes actores, así como en la recuperación de saberes ancestrales de T. pavonia y su articulación con el conocimiento científico, fortaleciendo los enlaces socio-eco sistémicos e identificación con la especie vegetal, permitiendo a su vez aprendizajes significativos que pueden llegar a contribuir con la conservación, manejo adecuado de la especie, potencialización en los diferentes usos y desarrollo local.

Se evidenció que los procesos participativos se afianzan en la medida que los diferentes actores se sienten identificados con la investigación, tienen un rol protagónico, no hay exclusión, se aceptan las diferentes opiniones y visiones; donde la participación no se asume como una simple consulta o información, sino que trasciende desde la formación de grupos de trabajo para responder a objetivos planteados hasta incidir en la toma de decisiones, que repercuten en la apropiación social del conocimiento, siendo relevante un estudio más amplio de T. pavonia al ser una especie silvestre de importancia económica y cultural.

\section{REFERENCIAS}

Arias, J.C. y Cárdenas, D., Manual de identificación, selección y evaluación de oferta de productos forestales no maderables (en la web: http://www.corpoica.org.co/sitioweb/archivos/publicaciones/pfnm.pdf, acceso: 18 de septiembre de 2015) Instituto Amazónico de Investigaciones Científicas- Sinchi (2007)

Delgado, M., y otros cinco autores. Local perceptions on social-ecological dynamics in Latin America in three community-based natural resource management systems, doi:10.5751/ES-07965-200424, Ecology \& Society (en línea), 20(4), 328-361(2015)

De Villota, C. Informe proyecto investiguemos la watsimba. Grupo de investigación "Exploradores". Institución Educativa Escuela Normal Superior de Putumayo, Sibundoy, Colombia (2010)

Domínguez, Z. Las estrategias didácticas y su relación con el aprendizaje de las ciencias sociales en los alumnos de primer año de secundaria de la I.E. Miguel Cortés de Castilla. Instituto De Investigación Y Promoción Para El Desarrollo, Universidad Nacional del Piura, Perú (2011)

Dubois, A., La construcción de lo público: Participación e inclusión, ALBOAN, 48(1), 3-6 (2008)

Endere, M., y Mercedes, M. Los conocimientos tradicionales y los desafíos de su protección legal en Argentina, Quinto Sol: Revista de historia regional, 17(2), 1-20 (2013)

Fals, O. Orígenes universales y retos actuales de la Investigación-Acción Participativa (en la web http://es.scribd.com/doc/12958462/Origenes-Universales-y-Retos-Actuales-de-La-IAP, acceso: 20 de enero de 2015) (2012)

Fernández, L. y Gutiérrez, M., Bienestar Social, Económico y Ambiental para las Presentes y Futuras Generaciones, doi: 10.4067/S0718-07642013000200013, Inf. Tecnol, 24(2), 121-130 (2013)

García, J.; Castillo, A. y J. Aguilera. Sociedad de conocimiento y políticas neoliberales: La escuela bajo acoso, Revista Latinoamericana de Estudios Educativos (en línea), 38 (1-2), 35-39 (2008)

Hatcher, J. Dialogue, consensus and vision Participatory and negotiated territorial development-more than a methodology - A strategy for territorial interaction and integration. Land Tenure Working Paper, FAO, (2009)

Holguin, M. T. y Lezaca, J. A. Una visión de la educación ambiental desde la perspectiva económica y empresarial. Los proyectos ambientales escolares (PRAE) y los planes integrales de gestión ambiental (En la web: http://www.slideshare.net/katherinnd15/una-visin-de-la-educacin-ambiental-ultima-versin, acceso: 19 de octubre del 2015), (2010)

León, A., Luna, G. y Leonel, H., Software educativo "mundo agroforestal": Estudio de caso, Subcuenca Alta del Río Pasto, Nariño, Colombia, http://revistas.udenar.edu.co/index.php/rfacia/article/view/1938/2325, ISSN: 0120-0135, Revista de Ciencias Agrícolas, 31(1), 65- 77 (2014) 
Leonel, H. y Luna, G. Herramientas para la formación agroforestal, 1ª edición, 124. Editorial Universitaria, , Pasto, Colombia (2016)

Leonel, H.; Aguilar, M; y H. Reyes, Factores sociodemográficos y niveles de participación en la gestión de la cuenca hidrográfica del Río valles, oriente de México, Prospectiva, 15(1), (2010)

Leonel, H. Gestión participativa de cuencas hidrográficas: El caso de la cuenca del río Valles, oriente de México. Tesis doctorado, Facultad de Ciencias Químicas, Ingeniería y Medicina; Programa Multidisciplinario de Posgrado en Ciencias Ambientales; Universidad Autónoma de San Luis Potosí, México (2011)

Leonel, H.; Luna, G. y Rivas, H. El juego como herramienta para la educación ambiental. 1ª edición, Agua para la vida, Pasto, Colombia (2013)

Linderoth, J., Why gamers don't learn more: An ecological approach to games as learning environments, doi: http://dx.doi.org/10.1386/jgvw.4.1.45_1, Journal of Gaming \& Virtual Worlds, 4(1), 45-62 (2012)

Martínez, M.A., y otros cuatro autores, Flora útil de los cafetales en la Sierra Norte de Puebla, México, Revista Mexicana de Biodiversidad: 78 (1), 15-40 (2007)

Paguatian, A. Caracterización ecológica y morfológica de Tigridia pavonia, en los municipios de Santiago y Sibundoy. Trabajo de grado Ingeniería Agroforestal, Facultad de Ciencias Agrícolas, Universidad de Nariño. Pasto- Colombia (2007)

Pérez, J., Pachón, F. y Parrado, A., Escuelas de Campo en papa y su aporte en la construcción de procesos organizativos en dos municipios colombianos, doi: http://dx.doi.org/10.17584/rcch.2015v9i2.4186, Revista Colombiana de Ciencias Hortícolas, 9(2), 301-312 (2015)

Piña, J., Vásquez, L., y A. Arzate, Variety discrimination of Tigridia pavonia (L.F.) DC. assesed by different length RAPD primers, Electronic Journal of Biotechnology, 13(4), 1-7 (2010a).

Piña, J y otros cuatro autores. Caracterización morfológica y molecular de nueve variedades botánicas de Tigridia pavonia (L.F.) DC., Agrociencia: 44(2),147-158 (2010b)

Rodríguez, D., Rodríguez, P. y Santana, A., Fundamentos del paradigma ecológico en las ciencias sociales, http://www.pasosonline.org/Publicados/10112/PS0112_15.pdf, ISSN: 1695-7121, PASOS. Revista de Turismo y Patrimonio Cultural, 10(1), 167-172 (2012)

Sánchez, L. y Del Pino, M., Una mirada a la participación comunitaria en el proceso de contraloría social, Paradigma, 29(2), 35 - 53, (2008)

Torregroza, E.; Llamas, J. y Borja, F., Diferencias entre actores sociales en el conocimiento y la percepción de la vegetación de la cuenca de la Ciénaga de la Virgen (Cartagena de Indias, Colombia), http://www.scielo.org.pe/pdf/ecol/v13n2/a04v13n2.pdf, ISSN: 1726-2216, Ecología Aplicada, 13(2), 97-108 (2014)

Urquiza, A. y Cadenas, H., Sistemas socio-ecológicos: elementos teóricos y conceptuales para la discusión en torno a vulnerabilidad hídrica, doi: 10.4000/orda.1774, L'Ordinaire des Amériques, 18(1), 1-18 (2015)

Vázquez, L. y otros cuatro autores, Morphological description of nine botanical varieties of Tigridia pavonia (L.F.), Ker. Gawl, J. Appl. Bot: 75(1), 14-19 (2001a)

Vázquez, L., Norman, T., y Corona, M., Oceloxochitl Tigridia pavonia (L.F.) DC. Colección: Ciencias Naturales y Exactas, Serie: Ciencias Agrícolas. Universidad Autónoma del Estado de México, 69 - 81, (2001b)

Villamayor, C., La comunicación como perspectiva y como dimensión de los procesos sociales. Una experiencia de participación en las Políticas Públicas, Unirevista, 1(3), 1-11 (2006) 
